\title{
Existence of solution for a quasilinear elliptic Neumann problem involving multiple critical exponents
}

Yuanxiao $\mathrm{Li}^{1 *}$ and Xiying Wang ${ }^{1}$

${ }^{*}$ Correspondence:

yxiaoli06@163.com

${ }^{1}$ College of Science, Henan

University of Technology,

Zhengzhou, P.R. China

\section{Springer}

\begin{abstract}
In this paper, we study the Neumann boundary value problem to a quasilinear elliptic equation with the critical Sobolev exponent and critical Hardy-Sobolev exponent, and prove the existence of nontrivial nonnegative solution by means of variational method.
\end{abstract}

MSC: 35B33; 35J20; 35J62; 35J75; 35J92

Keywords: Quasilinear elliptic equation; Nontrivial weak solution; Multiple critical exponents; Variational method

\section{Introduction and main results}

In this paper, we discuss the following quasilinear elliptic problem with critical Sobolev exponent and critical Hardy-Sobolev exponent:

$$
\begin{cases}-\Delta_{p} u+\lambda|u|^{p-2} u=Q(x)|u|^{p^{*}-2} u+P(x) \frac{|u|^{p^{*}(t)-2} u}{|x|^{t}}, & x \in \Omega, \\ |\nabla u|^{p-2} \frac{\partial u}{\partial v}=0, & x \in \partial \Omega \backslash\{0\},\end{cases}
$$

where $\Omega \subset R^{N}$ is a bounded domain with $\partial \Omega \in C^{2}, 0 \in \partial \Omega, \Delta_{p} u=\operatorname{div}\left(|\nabla u|^{p-2} \nabla u\right)$ is the $p$-Laplace operator, $1<p<N, p^{*}=\frac{N p}{N-p}, p^{*}(t)=\frac{p(N-t)}{N-p}(0 \leq t<p)$ is the so-called critical Hardy-Sobolev exponent, and $v$ denotes the unit outward normal vector with respect to $\partial \Omega, \lambda \in R$ is a parameter, the weight functions $Q(x), P(x)$ are continuous on $\bar{\Omega}$. Such problems arise in the theory of quasiregular and quasiconformal mapping or in the study of non-Newtonian fluids. In the latter case, the parameter $p$ is a characteristic of the medium. Media with $p>2$ are called dilatant fluids and those with $p<2$ are called pseudoplastics. If $p=2$, they are Newtonian fluids.

The study of semilinear elliptic problems with critical growth terms is one of hot spots in partial differential equations. In the case of $p=2$, problem (1.1) is transformed into the

(c) The Author(s) 2020. This article is licensed under a Creative Commons Attribution 4.0 International License, which permits use, sharing, adaptation, distribution and reproduction in any medium or format, as long as you give appropriate credit to the original author(s) and the source, provide a link to the Creative Commons licence, and indicate if changes were made. The images or other third party material in this article are included in the article's Creative Commons licence, unless indicated otherwise in a credit line to the material. If material is not included in the article's Creative Commons licence and your intended use is not permitted by statutory regulation or exceeds the permitted use, you will need to obtain permission directly from the copyright holder. To view a copy of this licence, visit http://creativecommons.org/licenses/by/4.0/. 
following semilinear elliptic problem:

$$
\begin{cases}-\Delta u+\lambda u=Q(x)|u|^{2^{*}-2} u+P(x) \frac{|u|^{*}(t)-2}{\left.|x|\right|^{t}}, & x \in \Omega, \\ \frac{\partial u}{\partial \nu}=0, & x \in \partial \Omega \backslash\{0\} .\end{cases}
$$

When functions $Q(x)=1, P(x)=0$, Comte and Knaap [1] established the existence of nontrivial solution for problem (1.2) by variational method, while Adimurthi et al. [2] proved the existence of a nonradial positive solution. Chabrowski and Willem [3] obtained the existence of least energy solutions by solving minimization problem corresponding to

$$
S_{\lambda}=\inf _{u \in H^{1}(\Omega), \int_{\Omega} Q(x)|u|^{*}} \mathrm{~d} x \neq 0 \quad \frac{\int_{\Omega}\left(|\nabla u|^{2}+\lambda u^{2}\right) \mathrm{d} x}{\left(\int_{\Omega} Q(x)|u|^{2^{*}} \mathrm{~d} x\right)^{\frac{2}{2^{*}}}}
$$

if the coefficient $Q(x)$ is nonnegative and Hölder continuous. Subsequently, Chabrowski [4] proved the existence of at least two solutions to (1.2) by the mountain pass principle if one of the weight functions changes sign. In the paper [5], the authors studied the following semilinear elliptic problem with Hardy-Sobolev exponent:

$$
\begin{cases}-\Delta u-\mu \frac{u}{|x|^{2}}=\frac{|u|^{2^{*}(t)}-2}{|x|^{t}} u+f(x, u), & x \in \Omega, \\ \frac{\partial u}{\partial \nu}+\alpha(x) u=0, & x \in \partial \Omega,\end{cases}
$$

they obtained the existence of positive solutions by the mountain pass lemma without $(P S)$-condition and the strong maximum principle. Other related results on the semilinear elliptic problems can be seen in [6-12] and the references therein.

As for the quasilinear elliptic problems with critical Sobolev or Hardy-Sobolev exponents, the existence and multiplicity of solutions have also been studied extensively. Abreu et al. [13] studied the following the nonhomogeneous Neumann boundary problem:

$$
\begin{cases}-\Delta_{p} u+\lambda u^{p-1}=u^{q}, & x \in \Omega, \\ u>0, & x \in \Omega, \\ |\nabla u|^{p-2} \frac{\partial u}{\partial v}=\varphi, & x \in \partial \Omega,\end{cases}
$$

where $p-1<q \leq p^{*}-1, \varphi \in C^{\alpha}(\bar{\Omega}), 0<\alpha<1, \varphi \not \equiv 0$. They proved that there exists a $\lambda^{*}>0$ such that problem (1.4) has at least two positive solutions if $\lambda>\lambda^{*}$, has at least one positive solution if $\lambda=\lambda^{*}$, and has no positive solution if $\lambda<\lambda^{*}$ relying on the lower and upper solutions method and variational approach. Subsequently, Deng and Jin [14] also obtained the existence of solutions to problem (1.4) if $u^{q}$ is replaced by $\frac{u^{q}}{|x|^{t}}$, where $p-1<q \leq p^{*}(t)-1,0 \leq t<p-1$. Li and Xia [15] studied the existence of multiple solutions for quasilinear Neumann problem with critical Sobolev exponent. With regard to the multiple critical exponents, Filippucci et al. [16] investigated the quasilinear elliptic problem involving multiple critical terms on the whole space and obtained the existence of positive solutions by using the existence of extremals of some Hardy-Sobolev type embedding. Li et al. [17] showed the existence and multiplicity of solutions to the quasilinear elliptic equations with Dirichlet boundary conditions and combined critical HardySobolev terms on bounded smooth domains by employing Ekeland's variational principle. 
Bhakta [18] studied the existence and multiplicity of sign-changing solutions to Dirichlet problem for semilinear elliptic equation involving critical Sobolev and Hardy-Sobolev exponents. For more relevant information as regards the corresponding problems, the interested reader may refer to [19-26].

However, as far as we know, there are few results of the Neumann boundary condition for quasilinear elliptic equations with the critical Sobolev exponent and critical HardySobolev exponent. Motivated by the results of the above-mentioned papers, in this paper we aim to show the existence of nontrivial nonnegative solution to problem (1.1) by the variational method. The special features of this problem are the following. Firstly, due to the lack of compactness of the embedding of $W^{1, p}(\Omega) \hookrightarrow L^{p^{*}}(\Omega)$ and $W^{1, p}(\Omega) \hookrightarrow$ $L^{p^{*}(t)}\left(\Omega,|x|^{-t}\right)$, we cannot use the standard variational argument directly. In order to overcome this difficulty and obtain the existence of solutions, we have to add restrictions on the weight functions $Q(x)$ and $P(x)$ to prove that the corresponding functional of problem (1.1) satisfies the $(P S)_{c}$-condition in a suitable range by the Lions concentration-compactness principle. Secondly, when $\lambda \leq 0$, the weight function $P(x)$ is allowed to change sign and the space $W^{1, p}(\Omega)$ is not suitable for our problem. In order to obtain the existence of solution, we have to introduce a suitable space. This result is an extension of a work by Li and Xia [15].

Throughout this paper, we define $Q^{M}=\max _{x \in \partial \Omega} Q(x), Q_{M}=\max _{x \in \bar{\Omega}} Q(x), Q_{m}=$ $\min _{x \in \bar{\Omega}} Q(x), P_{M}=\max _{x \in \bar{\Omega}} P(x)$, the main results of this paper are the following theorems.

Theorem 1.1 Suppose that $\lambda>0$, and the coefficients $Q(x), P(x)$ are positive continuous on $\bar{\Omega}$. Then there exists a constant $\lambda^{*}>0$ such that Problem (1.1) has at least one nontrivial nonnegative solution for $0<\lambda<\lambda^{*}$, where

$$
\begin{aligned}
\lambda^{*}= & |\Omega|^{-1} \max \left\{N^{\frac{p}{N}}\left(c^{*}\right)^{\frac{p}{N}}\left(\int_{\Omega} Q(x) \mathrm{d} x\right)^{\frac{N-p}{N}},\right. \\
& \left.\left(\frac{p(N-t)}{p-t}\right)^{\frac{p-t}{N-t}}\left(c^{*}\right)^{\frac{p-t}{N-t}}\left(\int_{\Omega} \frac{P(x)}{|x|^{t}} \mathrm{~d} x\right)^{\frac{N-p}{N-t}}\right\}, \\
c^{*}= & \min \left\{\frac{S^{\frac{N}{p}}}{N 2^{\frac{N+p}{p}} Q^{\frac{N-p}{p}}(0)}, \frac{(p-t) S_{H}^{\frac{N-t}{p-t}}}{p(N-t) 2^{\frac{N+p-2 t}{p-t}} P^{\frac{N-p}{p-t}}(0)}, \frac{S^{\frac{N}{p}}}{N Q_{M}^{\frac{N-p}{p}}}, \frac{S^{\frac{N}{p}}}{2 N Q^{M \frac{N-p}{p}}}\right\} .
\end{aligned}
$$

Theorem 1.2 Suppose that $\lambda>0$, the functions $Q(x), P(x)$ are positive continuous functions on $\bar{\Omega}, Q_{M} \leq 2^{\frac{p}{N-p}} Q^{M}, Q(0) \leq 2^{\frac{-N}{N-p}} Q^{M}, P(0) \leq\left(\frac{N(p-t))_{H}^{\frac{N-t}{p^{-t}}}}{p(N-t) S^{\frac{N}{p}} 2^{\frac{N-t}{p-t}}} Q^{M^{\frac{N-p}{p}}}\right)^{\frac{p-t}{N-p}}$. If there exists $y \in$ $\partial \Omega$ such that $Q^{M}=Q(y)$ and $|Q(y)-Q(x)|=o\left(|x-y|^{\sigma}\right)$ for $x \rightarrow y$, where $1<\sigma<\frac{N}{p-1}$, then Problem (1.1) has at least one nontrivial nonnegative solution for each $\lambda>0$ and $N>2 p-1$.

Theorem 1.3 Suppose that $\lambda \leq 0$, and the coefficients $Q(x), P(x)$ satisfy the following conditions:

(A1) $Q(x)$ is a positive continuous function on $\bar{\Omega}$;

(A2) $P(x)$ is a continuous and changing sign function on $\bar{\Omega}$, satisfying $\int_{\Omega} \frac{P(x)}{|x|^{t}} \mathrm{~d} x<0$.

Then there exists a constant $\mu^{*}>0$ such that Problem (1.1) has at least one nontrivial nonnegative solution for all $0 \leq \mu<\mu^{*}$, where $\mu=-\lambda$, and $\mu^{*}$ will be given in Sect. 4 . 
The outline of this paper is as follows. In Sect. 2, we present some necessary preliminaries. Section 3 is devoted to the proofs of Theorems 1.1 and 1.2. The proof of Theorem 1.3 is given in Sect. 4.

\section{Preliminaries}

Let $W^{1, p}(\Omega)$ be the Sobolev space with norm $\|u\|=\left(\int_{\Omega}\left(|\nabla u|^{p}+|u|^{p}\right) \mathrm{d} x\right)^{\frac{1}{p}}$, the best Sobolev constant and the best Hardy-Sobolev constant are defined by

$$
S=\inf _{D^{1, p}\left(R^{N}\right) \backslash\{0\}} \frac{\int_{R^{N}}|\nabla u|^{p} \mathrm{~d} x}{\left(\int_{R^{N}}|u|^{p^{*}} \mathrm{~d} x\right)^{\frac{p}{p^{*}}}}, \quad S_{H}=\inf _{D^{1, p}\left(R^{N}\right) \backslash\{0\}} \frac{\int_{R^{N}}|\nabla u|^{p} \mathrm{~d} x}{\int_{R^{N}}\left(\frac{|u|^{p^{*}(t)}}{|x|^{t}} \mathrm{~d} x\right)^{\frac{p}{p^{*}(t)}}},
$$

where $D^{1, p}\left(R^{N}\right)=\left\{\nabla u \in L^{p}\left(R^{N}\right), u \in L^{p^{*}}\left(R^{N}\right)\right\}$. The constant $S$ is achieved by the functional $u_{\varepsilon}$ given by

$$
u_{\varepsilon}(x)=C_{N p} \varepsilon^{\frac{N-p}{p^{2}}}\left(\varepsilon+|x-y|^{\frac{p}{p-1}}\right)^{\frac{p-N}{p}},
$$

where the constant $C_{N p}$ is chosen such that $-\Delta_{p} u_{\varepsilon}=\left|u_{\varepsilon}\right|^{p^{*}-1}$ in $R^{N}$.

Next, we give the definition of the weak solution to Problem (1.1).

Definition 2.1 A function $u \in W^{1, p}(\Omega)$ is said to be a weak solution of Problem (1.1) if it satisfies

$$
\begin{aligned}
& \int_{\Omega}|\nabla u|^{p-2} \nabla u \nabla \varphi \mathrm{d} x+\lambda \int_{\Omega}|u|^{p-2} u \varphi \mathrm{d} x \\
& \quad=\int_{\Omega} Q(x)|u|^{p^{*}-2} u \varphi \mathrm{d} x+\int_{\Omega} P(x) \frac{|u|^{p^{*}(t)-2} u}{|x|^{t}} \varphi \mathrm{d} x, \quad \forall \varphi \in W^{1, p}(\Omega) .
\end{aligned}
$$

The corresponding nonnegative solutions of Problem (1.1) are equivalent to the critical points of the energy functional

$$
J_{\lambda}(u)=\frac{1}{p} \int_{\Omega}|\nabla u|^{p} \mathrm{~d} x+\frac{\lambda}{p} \int_{\Omega}|u|^{p} \mathrm{~d} x-\frac{1}{p^{*}} \int_{\Omega} Q(x)|u|^{p^{*}} \mathrm{~d} x-\frac{1}{p^{*}(t)} \int_{\Omega} P(x) \frac{|u|^{p^{*}(t)}}{|x|^{t}} \mathrm{~d} x .
$$

In order to obtain the existence of solution to Problem (1.1), we need the following lemmas.

Lemma $2.1([13])$

(1) For $\varepsilon>0$ small enough and $N>2 p-1$, we have

$$
\begin{aligned}
& \int_{\Omega}\left|\nabla u_{\varepsilon}\right|^{p} \mathrm{~d} x=\frac{1}{2} \int_{R^{N}}\left|\nabla u_{\varepsilon}\right|^{p} \mathrm{~d} x-K_{1}(\varepsilon)+o\left(\varepsilon^{\frac{p-1}{p}}\right), \\
& \int_{\Omega}\left|u_{\varepsilon}\right|^{p^{*}} \mathrm{~d} x=\frac{1}{2} \int_{R^{N}}\left|u_{\varepsilon}\right|^{p^{*}} \mathrm{~d} x-K_{2}(\varepsilon)+o\left(\varepsilon^{\frac{p-1}{p}}\right),
\end{aligned}
$$

where $K_{1}(\varepsilon), K_{2}(\varepsilon)$ satisfy

$$
\lim _{\varepsilon \rightarrow 0} \varepsilon^{-\frac{p-1}{p}} K_{1}(\varepsilon)=\frac{1}{2} H(y) C_{N p}^{p}\left(\frac{N-p}{p-1}\right)^{p} \int_{R^{N-1}}\left(1+\left|z^{\prime}\right|^{\frac{p}{p-1}}\right)^{-N}\left|z^{\prime}\right|^{\frac{3 p-2}{p-1}} \mathrm{~d} z^{\prime}
$$




$$
\begin{aligned}
& =K_{1}, \\
\lim _{\varepsilon \rightarrow 0} \varepsilon^{-\frac{p-1}{p}} K_{2}(\varepsilon) & =\frac{1}{2} H(y) C_{N p}^{p^{*}} \int_{R^{N-1}}\left(1+\left|z^{\prime}\right|^{\frac{p}{p-1}}\right)^{-N}\left|z^{\prime}\right|^{2} \mathrm{~d} z^{\prime} \\
& =K_{2},
\end{aligned}
$$

where $H(y)=\frac{1}{N-1} \sum_{i-1}^{N-1} \alpha_{i}, \alpha_{i}(i=1,2, \ldots, N-1)$ are the principal curvatures of $\partial \Omega$ at $y$.

(2)

$$
\int_{\Omega}\left|u_{\varepsilon}\right|^{p} \mathrm{~d} x= \begin{cases}O\left(\varepsilon^{\frac{N-p}{p}}\right), & p<N<p^{2} \\ O\left(\varepsilon^{\frac{N-p}{p}}\right)|\ln \varepsilon|, & N=p^{2} \\ O\left(\varepsilon^{p-1}\right), & N>p^{2}\end{cases}
$$

Lemma 2.2 ([14] (Hardy-Sobolev inequality)) Assume that $1<p<N$ and $p^{*}(t)=\frac{p(N-t)}{N-p}$, $0 \leq t \leq p$. Then there exists a constant $C>0$ such that, for any $u \in W^{1, p}(\Omega)$,

$$
\int_{\Omega} \frac{|u|^{p^{*}(t)}}{|x|^{t}} \mathrm{~d} x \leq C\left(\int_{\Omega}\left(|\nabla u|^{p}+|u|^{p}\right) \mathrm{d} x\right)^{\frac{p^{*}(t)}{p}} .
$$

By Sobolev's inequality, there exists a constant $C_{1}>0$ such that

$$
\int_{\Omega}|u|^{p^{*}} \mathrm{~d} x \leq C_{1}\|u\|^{p^{*}} \quad \text { for } u \in W^{1, p}(\Omega) .
$$

Now, we prove that the energy functional $J_{\lambda}(u)$ satisfies the geometry of mountain pass lemma.

Lemma 2.3 Assume that coefficients $Q(x), P(x)$ are positive continuous functions on $\bar{\Omega}$, then for each $\lambda>0$, we have

(i) there exist constants $\beta, \rho>0$ such that $J_{\lambda}(u) \geq \beta$ for $\|u\|=\rho$;

(ii) there exists $u_{0} \in W^{1, p}(\Omega)$ such that $J_{\lambda}\left(u_{0}\right)<0$ and $\left\|u_{0}\right\|>\rho$.

Proof (i) From (2.3) and (2.4), we know that

$$
\begin{aligned}
J_{\lambda}(u) & \geq \frac{1}{p} \min \{1, \lambda\}\|u\|^{p}-\frac{Q_{M}}{p^{*}} \int_{\Omega}|u|^{p^{*}} \mathrm{~d} x-\frac{P_{M}}{p^{*}(t)} \int_{\Omega} \frac{|u|^{p^{*}(t)}}{|x|^{t}} \mathrm{~d} x \\
& \geq \frac{1}{p} \min \{1, \lambda\}\|u\|^{p}-\frac{C_{1} Q_{M}}{p^{*}}\|u\|^{p^{*}}-\frac{C P_{M}}{p^{*}(t)}\|u\|^{p^{*}(t)} .
\end{aligned}
$$

Since $p<p^{*}(t)<p^{*}$, then there exist constants $\rho, \beta>0$ such that $J_{\lambda}(u) \geq \beta$ for $u \in \partial B_{\rho}=$ $\left\{u \in W^{1, p}(\Omega),\|u\|=\rho\right\}$.

(ii) Choosing $u \in W^{1, p}(\Omega)$ and $u \neq \equiv$, we have

$$
\begin{aligned}
J_{\lambda}(\tau u)= & \frac{\tau^{p}}{p} \int_{\Omega}\left(|\nabla u|^{p} \mathrm{~d} x+\lambda|u|^{p}\right) \mathrm{d} x-\frac{\tau^{p^{*}}}{p^{*}} \int_{\Omega} Q(x)|u|^{p^{*}} \mathrm{~d} x \\
& -\frac{\tau^{p^{*}(t)}}{p^{*}(t)} \int_{\Omega} \frac{P(x)|u|^{p^{*}(t)}}{|x|^{t}} \mathrm{~d} x, \quad \tau>0 .
\end{aligned}
$$


Because of $\lim _{\tau \rightarrow+\infty} J_{\lambda}(\tau u)=-\infty$, there exists $\tau_{0}>0$ such that $\left\|\tau_{0} u\right\|>\rho$ and $J_{\lambda}\left(\tau_{0} u\right)<0$. Let $u_{0}=\tau_{0} u$, then condition (ii) holds. The proof of Lemma 2.3 is completed.

We define

$$
\Gamma=\left\{h \in C([0,1]), W^{1, p}(\Omega) \mid h(0)=0, h(1)=\tau_{0} u=u_{0}\right\}, \quad c=\inf _{h \in \Gamma} \sup _{\tau \in[0,1]} J_{\lambda}(h(\tau)) .
$$

Using Lemma 2.3 and the mountain pass lemma, there exists a $(P S)_{c}$-sequence $\left\{u_{n}\right\} \subset$ $W^{1, p}(\Omega)$ such that $J_{\lambda}\left(u_{n}\right) \rightarrow c, J_{\lambda}^{\prime}\left(u_{n}\right) \rightarrow 0$ as $n \rightarrow \infty$. Then we have the following lemma.

Lemma 2.4 Assume that $\lambda>0$, and the coefficients $Q(x), P(x)$ are positive continuous on $\bar{\Omega}$. Then the energy functional $J_{\lambda}(u)$ satisfies the $(P S)_{c}$ condition for $c<c^{*}$, where $c^{*}=\min \left\{\frac{S^{\frac{N}{p}}}{N Q_{M}^{\frac{N-p}{p}}}, \frac{S^{\frac{N}{p}}}{2 N Q^{M \frac{N-p}{p}}}, \frac{S^{\frac{N}{p}}}{N 2^{\frac{N+p}{p}} Q^{\frac{N-p}{p}}(0)}, \frac{(p-t) S_{H}^{\frac{N-t}{p-t}}}{p(N-t) 2^{\frac{N+p-2 t}{p-t}} P^{\frac{N-p}{p-t}}(0)}\right\}$.

Proof Let $\left\{u_{n}\right\} \subset W^{1, p}(\Omega)$ be a $(P S)_{c}$ sequence for $J_{\lambda}(u)$ with $c<c^{*}$, firstly, we prove that $\left\{u_{n}\right\}$ is bounded. Since $J_{\lambda}\left(u_{n}\right) \rightarrow c, J_{\lambda}^{\prime}\left(u_{n}\right) \rightarrow 0$ as $n \rightarrow \infty$, we have

$$
\begin{aligned}
J_{\lambda}\left(u_{n}\right)= & \frac{1}{p} \int_{\Omega}\left|\nabla u_{n}\right|^{p} \mathrm{~d} x+\frac{\lambda}{p} \int_{\Omega}\left|u_{n}\right|^{p} \mathrm{~d} x-\frac{1}{p^{*}} \int_{\Omega} Q(x)\left|u_{n}\right|^{p^{*}} \mathrm{~d} x \\
& \quad-\frac{1}{p^{*}(t)} \int_{\Omega} P(x) \frac{\left|u_{n}\right|^{p^{*}(t)}}{|x|^{t}} \mathrm{~d} x \\
= & c+o(1), \\
\left\langle J_{\lambda}^{\prime}\left(u_{n}\right), u_{n}\right\rangle= & \int_{\Omega}\left|\nabla u_{n}\right|^{p} \mathrm{~d} x+\lambda \int_{\Omega}\left|u_{n}\right|^{p} \mathrm{~d} x-\int_{\Omega} Q(x)\left|u_{n}\right|^{p^{*}} \mathrm{~d} x \\
& -\int_{\Omega} P(x) \frac{\left|u_{n}\right|^{p^{*}(t)}}{|x|^{t}} \mathrm{~d} x \\
= & o(1) .
\end{aligned}
$$

Combining (2.5) with (2.6), it follows from the property of function $Q(x)$ that

$$
\begin{aligned}
c+o(1)= & J_{\lambda}\left(u_{n}\right)-\frac{1}{p^{*}(t)}\left\langle J_{\lambda}^{\prime}\left(u_{n}\right), u_{n}\right\rangle \\
= & \left(\frac{1}{p}-\frac{1}{p^{*}(t)}\right) \int_{\Omega}\left(\left|\nabla u_{n}\right|^{p} \mathrm{~d} x+\lambda\left|u_{n}\right|^{p}\right) \\
& +\left(\frac{1}{p^{*}(t)}-\frac{1}{p^{*}}\right) \int_{\Omega} Q(x)\left|u_{n}\right|^{p^{*}} \mathrm{~d} x \\
\geq & \left(\frac{1}{p}-\frac{1}{p^{*}(t)}\right) \int_{\Omega}\left(\left|\nabla u_{n}\right|^{p}+\lambda\left|u_{n}\right|^{p}\right) \mathrm{d} x,
\end{aligned}
$$

thus, we can get that $\left\{u_{n}\right\}$ is bounded in $W^{1, p}(\Omega)$.

Next, we prove that $\left\{u_{n}\right\}$ is relatively compact in $W^{1, p}(\Omega)$. Since $\left\{u_{n}\right\}$ is bounded in $W^{1, p}(\Omega)$, we know that there exists a subsequence, still denoted by $\left\{u_{n}\right\}$, such that

$$
u_{n} \rightarrow u \quad \text { weakly in } W^{1, p}(\Omega),
$$




$$
\begin{array}{ll}
u_{n} \rightarrow u & \text { weakly in } L^{p^{*}}(\Omega), \\
u_{n} \rightarrow u & \text { weakly in } L^{p^{*}(t)}\left(\Omega,|x|^{-t}\right), \\
u_{n} \rightarrow u & \text { strongly in } L^{p}(\Omega), \\
u_{n} \rightarrow u & \text { almost everywhere in } \Omega .
\end{array}
$$

By the Lions concentration-compactness principle [27], there exist at most countable set $J$, a set of distinct points $\left\{x_{j}\right\}_{j \in J} \subset \bar{\Omega} \backslash\{0\}$, sets of nonnegative real numbers $\left\{\mu_{j}\right\}_{j \in J},\left\{v_{j}\right\}_{j \in J}$ and nonnegative real numbers $\mu_{0}, v_{0}, \gamma_{0}$ such that

$$
\begin{aligned}
& \left|\nabla u_{n}\right|^{p} \mathrm{~d} x \rightarrow \mathrm{d} \mu \geq|\nabla u|^{p} \mathrm{~d} x+\sum_{j \in J} \mu_{j} \delta_{x_{j}}+\mu_{0} \delta_{0} \\
& \left|u_{n}\right|^{p^{*}} \mathrm{~d} x \rightarrow \mathrm{d} v=|u|^{p^{*}} \mathrm{~d} x+\sum_{j \in J} v_{j} \delta_{x_{j}}+v_{0} \delta_{0} \\
& \frac{\left|u_{n}\right|^{p^{*}(t)}}{|x|^{t}} \mathrm{~d} x \rightarrow \mathrm{d} \gamma=\frac{|u|^{p^{*}(t)}}{|x|^{t}} \mathrm{~d} x+\gamma_{0} \delta_{0}
\end{aligned}
$$

in the weak sense of measure, where $\delta_{x}$ is the Dirac mass at $x$, and the constants $\mu_{j}, v_{j}, \gamma_{0}$ satisfying

$$
\begin{aligned}
& S v_{j}^{\frac{p}{p^{*}}} \leq \mu_{j}, \quad \text { where } x_{j} \in \Omega, j \in J, \\
& 2^{-\frac{p}{N}} S v_{j}^{\frac{p}{p^{*}}} \leq \mu_{j}, \quad \text { where } x_{j} \in \partial \Omega, j \in J \cup\{0\}, \\
& 2^{-\frac{p-t}{N-t}} S_{H} \gamma_{0}^{\frac{p}{p^{*}(t)}} \leq \mu_{0} .
\end{aligned}
$$

Now, we prove $\mu_{j}=0$ and $v_{j}=0$, where $j \in J$. In fact, we choose $\varepsilon>0$ sufficiently small such that $0 \notin B_{\varepsilon}\left(x_{j}\right)$ and $B_{\varepsilon}\left(x_{i}\right) \cap B_{\varepsilon}\left(x_{j}\right)=\varnothing$ for $i \neq j, i, j \in J$. Let $\phi_{\varepsilon}^{j}(x)$ be a smooth cut-off function centered at $x_{j}$ such that

$$
0 \leq \phi_{\varepsilon}^{j}(x) \leq 1 \quad \text { for }\left|x-x_{j}\right|<\varepsilon, \quad \phi_{\varepsilon}^{j}(x)=\left\{\begin{array}{ll}
1, & \left|x-x_{j}\right| \leq \frac{\varepsilon}{2}, \\
0, & \left|x-x_{j}\right| \geq \varepsilon,
\end{array} \quad \text { and } \quad\left|\nabla \phi_{\varepsilon}^{j}\right| \leq \frac{4}{\varepsilon}\right.
$$

Noting that

$$
\begin{aligned}
& \left\langle J_{\lambda}^{\prime}\left(u_{n}\right), u_{n} \phi_{\varepsilon}^{j}(x)\right\rangle \\
& =\int_{\Omega}\left|\nabla u_{n}\right|^{p} \phi_{\varepsilon}^{j}(x) \mathrm{d} x+\int_{\Omega}\left|\nabla u_{n}\right|^{p-2} \nabla u_{n} \nabla \phi_{\varepsilon}^{j}(x) u_{n} \mathrm{~d} x+\lambda \int_{\Omega}\left|u_{n}\right|^{p} \phi_{\varepsilon}^{j}(x) \mathrm{d} x \\
& \quad-\int_{\Omega} Q(x)\left|u_{n}\right|^{p^{*}} \phi_{\varepsilon}^{j}(x) \mathrm{d} x-\int_{\Omega} P(x) \frac{\left|u_{n}\right|^{p^{*}(t)}}{|x|^{t}} \phi_{\varepsilon}^{j}(x) \mathrm{d} x,
\end{aligned}
$$

and by (2.7), we have that

$$
\begin{aligned}
& \lim _{\varepsilon \rightarrow 0} \lim _{n \rightarrow \infty} \int_{\Omega}\left|\nabla u_{n}\right|^{p} \phi_{\varepsilon}^{j}(x) \mathrm{d} x \geq \mu_{j}, \\
& \lim _{\varepsilon \rightarrow 0} \lim _{n \rightarrow \infty} \int_{\Omega}\left|\nabla u_{n}\right|^{p-2} \nabla u_{n} \nabla \phi_{\varepsilon}^{j}(x) u_{n} \mathrm{~d} x=0,
\end{aligned}
$$




$$
\begin{aligned}
& \lim _{\varepsilon \rightarrow 0} \lim _{n \rightarrow \infty} \int_{\Omega}\left|u_{n}\right|^{p} \phi_{\varepsilon}^{j}(x) \mathrm{d} x=0, \\
& \lim _{\varepsilon \rightarrow 0} \lim _{n \rightarrow \infty} \int_{\Omega} Q(x)\left|u_{n}\right|^{p^{*}} \phi_{\varepsilon}^{j}(x) \mathrm{d} x=Q\left(x_{j}\right) v_{j}, \\
& \lim _{\varepsilon \rightarrow 0} \lim _{n \rightarrow \infty} \int_{\Omega} P(x) \frac{\left|u_{n}\right|^{p^{*}(t)}}{|x|^{t}} \phi_{\varepsilon}^{j}(x) \mathrm{d} x=0 .
\end{aligned}
$$

Thus,

$$
0=\lim _{\varepsilon \rightarrow 0} \lim _{n \rightarrow \infty}\left\langle J_{\lambda}^{\prime}\left(u_{n}\right), u_{n} \phi_{\varepsilon}^{j}(x)\right\rangle \geq \mu_{j}-Q\left(x_{j}\right) v_{j} .
$$

If $v_{j} \neq 0$, by (2.8) and (2.9), we find that

$$
\begin{aligned}
& v_{j} \geq \frac{S^{\frac{N}{p}}}{Q^{\frac{N}{p}}\left(x_{j}\right)}, \quad x_{j} \in \Omega, \\
& v_{j} \geq \frac{S^{\frac{N}{p}}}{2 Q^{\frac{N}{p}}\left(x_{j}\right)}, \quad x_{j} \in \partial \Omega .
\end{aligned}
$$

On the other hand,

$$
\begin{aligned}
c= & \frac{1}{N} \int_{\Omega} Q(x)|u|^{p^{*}} \mathrm{~d} x+\frac{p-t}{p(N-t)} \int_{\Omega} P(x) \frac{|u|^{p^{*}(t)}}{|x|^{t}} \mathrm{~d} x \\
& +\frac{1}{N} \sum_{j \in J} Q\left(x_{j}\right) v_{j}+\frac{1}{N} Q(0) v_{0}+\frac{p-t}{p(N-t)} P(0) \gamma_{0},
\end{aligned}
$$

this implies $c \geq \frac{S^{\frac{N}{p}}}{N Q_{M}^{\frac{N-p}{p}}}$ or $c \geq \frac{S^{\frac{N}{p}}}{2 N Q^{\frac{N-p}{N}}}$, which is a contradiction. Hence, $\mu_{j}=v_{j}=0$.

Next, we consider the possibility of concentration at the origin. Let $\varepsilon>0$ be small enough such that $x_{j} \notin B_{\varepsilon}(0), j \in J$, choose a smooth cut-off function $\phi_{\varepsilon}^{0}(x)$ such that

$$
0 \leq \phi_{\varepsilon}^{0}(x) \leq 1 \quad \text { for }|x|<\varepsilon, \quad \phi_{\varepsilon}^{0}(x)=\left\{\begin{array}{ll}
1, & |x| \leq \frac{\varepsilon}{2}, \\
0, & |x| \geq \varepsilon,
\end{array} \quad \text { and } \quad\left|\nabla \phi_{\varepsilon}^{0}\right| \leq \frac{4}{\varepsilon}\right.
$$

Similarly, we get $\mu_{0} \leq Q(0) v_{0}+P(0) \gamma_{0}$. If $\mu_{0} \neq 0$, we have

$$
\frac{\mu_{0}}{2} \leq Q(0) v_{0} \quad \text { or } \quad \frac{\mu_{0}}{2} \leq P(0) \gamma_{0}
$$

using the above inequalities and combining (2.8) with (2.10), we can deduce that

$$
\begin{aligned}
& c \geq \frac{1}{N} Q(0) \nu_{0} \geq \frac{S^{\frac{N}{p}}}{2^{\frac{N+p}{p}} N Q^{\frac{N-p}{p}}(0)} \\
& \text { or } \quad c \geq \frac{p-t}{p(N-t)} P(0) \gamma_{0} \geq \frac{p-t}{p(N-t)} \frac{S^{\frac{N-t}{p-t}}}{2^{\frac{N+p-2 t}{p-t}} P^{\frac{N-p}{p-t}}(0)},
\end{aligned}
$$

which is a contradiction with the condition $c<c^{*}$. Hence, $\mu_{0}=v_{0}=\gamma_{0}=0$ and $u_{n} \rightarrow u$ strongly in $W^{1, p}(\Omega), \frac{\left|u_{n}\right|^{* *(t)}}{|x|^{t}} \rightarrow \frac{|u|^{* *(t)}}{|x|^{t}}$ strongly in $L^{p^{*}(t)}(\Omega)$. 


\section{Proofs of Theorems 1.1 and $\mathbf{1 . 2}$}

In order to prove Theorems 1.1 and 1.2, we also need the following lemma.

Lemma 3.1 Assume that $\lambda>0$ and the coefficients $Q(x), P(x)$ are positive continuous functions on $\bar{\Omega}$, then there exists $\lambda^{*}>0$ such that $\sup _{\tau \geq 0} J_{\lambda}(\tau u)<c^{*}$ for $0<\lambda<\lambda^{*}$.

Proof For $u \in W^{1, p}(\Omega) \backslash\{0\}$, we consider the functional

$$
\begin{aligned}
J_{\lambda}(\tau u)= & \frac{\tau^{p}}{p} \int_{\Omega}\left(|\nabla u|^{p}+\lambda|u|^{p}\right) \mathrm{d} x-\frac{\tau^{p *}}{p^{*}} \int_{\Omega} Q(x)|u|^{p^{*}} \mathrm{~d} x-\frac{\tau^{p^{*}(t)}}{p^{*}(t)} \int_{\Omega} P(x) \frac{|u|^{p^{*}(t)}}{|x|^{t}} \mathrm{~d} x \\
\leq & \sup _{\tau \geq 0}\left[\frac{\tau^{p}}{p} \int_{\Omega}\left(|\nabla u|^{p}+\lambda|u|^{p}\right) \mathrm{d} x\right. \\
& \left.-\frac{\tau^{p *}}{p^{*}} \int_{\Omega} Q(x)|u|^{p^{*}} \mathrm{~d} x-\frac{\tau^{p^{*}(t)}}{p^{*}(t)} \int_{\Omega} P(x) \frac{|u|^{p^{*}(t)}}{|x|^{t}} \mathrm{~d} x\right] \\
\leq & \frac{1}{N} \frac{\left(\int_{\Omega}\left(|\nabla u|^{p}+\lambda|u|^{p}\right) \mathrm{d} x\right)^{\frac{N}{p}}}{\left(\int_{\Omega} Q(x)|u|^{p^{*}} \mathrm{~d} x\right)^{\frac{N-p}{p}}}
\end{aligned}
$$

Choosing $u=A$ (constant) $\neq 0$, we find that

$$
\sup _{\tau \geq 0} J_{\lambda}(\tau A) \leq \frac{1}{N} \frac{\lambda^{\frac{N}{p}}|\Omega|^{\frac{N}{p}}}{\left(\int_{\Omega} Q(x) \mathrm{d} x\right)^{\frac{N-p}{p}}}
$$

which implies $\sup _{\tau \geq 0} J_{\lambda}(\tau A)<c^{*}$ for $0<\lambda<|\Omega|^{-1} N^{\frac{p}{N}}\left(c^{*}\right)^{\frac{p}{N}}\left(\int_{\Omega} Q(x) \mathrm{d} x\right)^{\frac{N-p}{N}}$.

Similarly,

$$
\sup _{\tau \geq 0} J_{\lambda}(\tau A) \leq \frac{p-t}{p(N-t)} \frac{\lambda^{\frac{N-t}{p-t}}|\Omega|^{\frac{N-t}{p-t}}}{\left(\int_{\Omega} \frac{P(x)}{|x|^{t}} \mathrm{~d} x\right)^{\frac{N-p}{p-t}}}<c^{*}
$$

for $0<\lambda<|\Omega|^{-1}\left(\frac{p(N-t)}{p-t}\right)^{\frac{p-t}{N-t}}\left(c^{*}\right)^{\frac{p-t}{N-t}}\left(\int_{\Omega} \frac{P(x)}{|x|^{t}} \mathrm{~d} x\right)^{\frac{N-p}{N-t}}$.

Set $\lambda^{*}=|\Omega|^{-1} \max \left\{N \frac{p}{N}\left(c^{*}\right)^{\frac{p}{N}}\left(\int_{\Omega} Q(x) \mathrm{d} x\right)^{\frac{N-p}{N}},\left(\frac{p(N-t)}{p-t}\right)^{\frac{p-t}{N-t}}\left(c^{*}\right)^{\frac{p-t}{N-t}}\left(\int_{\Omega} \frac{P(x)}{|x|^{t}} \mathrm{~d} x\right)^{\frac{N-p}{N-t}}\right\}$, then we have $\sup _{\tau \geq 0} J_{\lambda}(\tau A)<c^{*}$ for $0<\lambda<\lambda^{*}$, and

$$
0<\beta \leq c=\inf _{h \in \Gamma} \max _{\tau \in[0,1]} J_{\lambda}(h(\tau)) \leq \max _{\tau \geq 0} J_{\lambda}(\tau A)<c^{*} .
$$

Proof of Theorem 1.1 Applying Lemma 2.4, we know that the functional $J_{\lambda}(u)$ satisfies the $(P S)_{c}$-condition, by Lemma 3.1 and the mountain pass theorem, we obtain that Problem (1.1) has at least one nontrivial solution $u$. On the other hand, since $J_{\lambda}(u)=J_{\lambda}(|u|)$, then Problem (1.1) has at least one nontrivial nonnegative solution. The proof of Theorem 1.1 is completed.

In order to prove Theorem 1.2, we also need the following lemma.

Lemma 3.2 Suppose that $\lambda>0$ and the coefficients $Q(x), P(x)$ are positive continuous functions on $\bar{\Omega}, c^{*}=\frac{S^{\frac{N}{p}}}{2 N Q^{\frac{N-p}{p}}}$. If there exists $y \in \partial \Omega$ such that $Q^{M}=Q(y)$ and $|Q(y)-Q(x)|=$ 
$o\left(|x-y|^{\sigma}\right)$ for $x \rightarrow y$, where $1<\sigma<\frac{N}{p-1}$. Then, for each $\lambda>0$ and $N>2 p-1$, there exists $a$ nonnegative function $v \in W^{1, p}(\Omega)$ and $v \neq \equiv$ such that

$$
\sup _{\tau \geq 0} J_{\lambda}(\tau v)<c^{*}
$$

Proof Firstly, we consider the functional

$$
\begin{aligned}
g(\tau)= & J_{\lambda}\left(\tau u_{\varepsilon}\right)=\frac{\tau^{p}}{p} \int_{\Omega}\left(\left|\nabla u_{\varepsilon}\right|^{p}+\lambda\left|u_{\varepsilon}\right|^{p}\right) \mathrm{d} x-\frac{\tau^{p *}}{p^{*}} \int_{\Omega} Q(x)\left|u_{\varepsilon}\right|^{p^{*}} \mathrm{~d} x \\
& -\frac{\tau^{p *(t)}}{p^{*}(t)} \int_{\Omega} P(x) \frac{\left|u_{\varepsilon}\right|^{p^{*}(t)}}{|x|^{t}} \mathrm{~d} x, \quad \tau>0 .
\end{aligned}
$$

Since $\lim _{\tau \rightarrow \infty} g(\tau)=-\infty, g(0)=0$, and $g(\tau)>0$ for $\tau \rightarrow 0^{+}$, we know that there exists some $\tau_{\varepsilon}>0$ such that

$$
\begin{aligned}
g\left(\tau_{\varepsilon}\right)= & \sup _{\tau \geq 0} J_{\lambda}\left(\tau u_{\varepsilon}\right)=\frac{\tau_{\varepsilon}^{p}}{p} \int_{\Omega}\left(\left|\nabla u_{\varepsilon}\right|^{p}+\lambda\left|u_{\varepsilon}\right|^{p}\right) \mathrm{d} x-\frac{\tau_{\varepsilon}^{p *}}{p^{*}} \int_{\Omega} Q(x)\left|u_{\varepsilon}\right|^{p^{*}} \mathrm{~d} x \\
& -\frac{\tau_{\varepsilon}^{p *(t)}}{p^{*}(t)} \int_{\Omega} P(x) \frac{\left|u_{\varepsilon}\right|^{p^{*}(t)}}{|x|^{t}} \mathrm{~d} x \\
\leq & \frac{1}{N}\left[\frac{\int_{\Omega}\left|\nabla u_{\varepsilon}\right|^{p} \mathrm{~d} x}{\left(\int_{\Omega} Q(x)\left|u_{\varepsilon}\right|^{p^{*}} \mathrm{~d} x\right)^{\frac{N-p}{N}}}\right]^{\frac{N}{p}}+\lambda \frac{\tau_{\varepsilon}^{p}}{p} \int_{\Omega}\left|u_{\varepsilon}\right|^{p} \mathrm{~d} x
\end{aligned}
$$

where $\tau_{\varepsilon}$ is uniformly bounded for $\varepsilon>0$ sufficiently small.

Next, we prove that $\int_{\Omega} Q(x)\left|u_{\varepsilon}\right|^{p^{*}} \mathrm{~d} x=Q^{M} \int_{\Omega}\left|u_{\varepsilon}\right|^{p^{*}} \mathrm{~d} x+o\left(\varepsilon^{\frac{p-1}{p}}\right)$.

Since $|Q(x)-Q(y)|=o\left(|x-y|^{\sigma}\right)$ for $x \rightarrow y$, then there exists $\delta>0$ such that $|Q(x)-Q(y)| \leq$ $C_{2}|x-y|^{\sigma}$ for $|x-y|<\delta$, where $C_{2}>0$ is constant. Moreover, a series of computations yield

$$
\begin{aligned}
& \int_{\Omega}|Q(x)-Q(y)|\left|u_{\varepsilon}\right|^{p^{*}} \mathrm{~d} x \\
& \quad \leq \int_{\Omega \cap|x-y| \leq \delta}|Q(x)-Q(y)|\left|u_{\varepsilon}\right|^{p^{*}} \mathrm{~d} x+\int_{\Omega \cap|x-y| \geq \delta}|Q(x)-Q(y)|\left|u_{\varepsilon}\right|^{p^{*}} \mathrm{~d} x \\
& \quad \leq C_{2} \int_{|x-y| \leq \delta}|x-y|^{\sigma}\left|u_{\varepsilon}\right|^{p^{*}} \mathrm{~d} x+2 Q_{M} \int_{\Omega \cap|x-y| \geq \delta}\left|u_{\varepsilon}\right|^{p^{*}} \mathrm{~d} x \\
& \quad=O\left(\varepsilon^{\frac{(p-1) \sigma}{p}}\right)+O\left(\varepsilon^{\frac{N}{p}}\right) .
\end{aligned}
$$

Noting that $N>2 p-1,1<\sigma<\frac{N}{p-1}$, then we have

$$
\int_{\Omega} Q(x)\left|u_{\varepsilon}\right|^{p^{*}} \mathrm{~d} x=Q^{M} \int_{\Omega}\left|u_{\varepsilon}\right|^{p^{*}} \mathrm{~d} x+o\left(\varepsilon^{\frac{p-1}{p}}\right) .
$$

Finally, we prove $\sup _{\tau \geq 0} J_{\lambda}\left(\tau u_{\varepsilon}\right)<c^{*}$. According to (3.1), (3.2), and Lemma 2.1, we have

$$
\sup _{\tau \geq 0} J_{\lambda}\left(\tau u_{\varepsilon}\right) \leq \frac{S^{\frac{N}{p}}}{2 N\left(Q^{M}\right)^{\frac{N-p}{p}}}\left[1+\frac{N-p}{p} M_{2}^{-1} K_{2}(\varepsilon)-\frac{N}{p} M_{1}^{-1} K_{1}(\varepsilon)\right]+o\left(\varepsilon^{\frac{p-1}{p}}\right),
$$

where $M_{1}=\frac{1}{2} \int_{R}\left|\nabla u_{\varepsilon}\right|^{p} \mathrm{~d} x, M_{2}=\frac{1}{2} \int_{R}\left|u_{\varepsilon}\right|^{p^{*}} \mathrm{~d} x$. 
Now, we claim that

$$
\lim _{\varepsilon \rightarrow 0} \varepsilon^{-\frac{p-1}{p}}\left(\frac{N-p}{p} M_{2}^{-1} K_{2}(\varepsilon)-\frac{N}{p} M_{1}^{-1} K_{1}(\varepsilon)\right)<0,
$$

which implies $\sup _{\tau \geq 0} J_{\lambda}(\tau v)<c^{*}$ holds. According to

$$
\begin{aligned}
& \lim _{\varepsilon \rightarrow 0} \varepsilon^{-\frac{p-1}{p}} K_{1}(\varepsilon)=K_{1}, \\
& \lim _{\varepsilon \rightarrow 0} \varepsilon^{-\frac{p-1}{p}} K_{1}(\varepsilon)=K_{2},
\end{aligned}
$$

we know that (3.3) is equivalent to $\frac{N-p}{N} \frac{M_{1}}{M_{2}}<\frac{K_{1}}{K_{2}}$.

From the expressions of $K_{1}, K_{2}, M_{1}, M_{2}$, and $u_{\varepsilon}$, a series of computations yield

$$
\begin{aligned}
\frac{K_{1}}{K_{2}}= & \frac{\frac{1}{2} H(y) C_{N p}^{p}\left(\frac{N-p}{p-1}\right)^{p} \int_{R^{N-1}}\left(1+\left|y^{\prime}\right|^{\frac{p}{p-1}}\right)^{-N}\left|y^{\prime}\right|^{\frac{3 p-2}{p-1}} \mathrm{~d} y^{\prime}}{\frac{1}{2} H(y) C_{N p}^{p^{*}} \int_{R^{N-1}}\left(1+\left|y^{\prime}\right|^{\frac{p}{p-1}}\right)^{-N}\left|y^{\prime}\right|^{2} \mathrm{~d} y^{\prime}} \\
= & C_{N p}^{p-p^{*}}\left(\frac{N-p}{p-1}\right)^{p} \frac{\int_{0}^{+\infty}\left(1+r^{\frac{p}{p-1}}\right)^{-N} r^{\frac{3 p-2}{p-1}+N-2} \mathrm{~d} r}{\int_{0}^{+\infty}\left(1+r^{\frac{p}{p-1}}\right)^{-N} r^{N} \mathrm{~d} r} \\
=C_{N p}^{p-p^{*}} & \left(\frac{N-p}{p-1}\right)^{p} \frac{\int_{0}^{+\infty}\left(1+r^{2}\right)^{-N} r^{\frac{2 N p+3 p-2 N-2}{p}} \mathrm{~d} r}{\int_{0}^{+\infty}\left(1+r^{2}\right)^{-N} r^{\frac{2 N p+p-2 N-2}{p}} \mathrm{~d} r}, \\
\frac{N-p) M_{1}}{N M_{2}} & =\frac{N-p}{N} C_{N p}^{p-p^{*}}\left(\frac{N-p}{p-1}\right)^{p} \frac{\int_{0}^{+\infty}\left(1+r^{\frac{p}{p-1}}\right)^{-N} r^{\frac{p}{p-1}+N-1} \mathrm{~d} r}{\int_{0}^{+\infty}\left(1+r^{\frac{p}{p-1}}\right)^{-N} r^{N-1} \mathrm{~d} r} \\
& =\frac{N-p}{N} C_{N p}^{p-p^{*}}\left(\frac{N-p}{p-1}\right)^{p} \frac{\int_{0}^{+\infty}\left(1+r^{2}\right)^{-N} r^{\frac{2 N p+p-2 N}{p}} \mathrm{~d} r}{\int_{0}^{+\infty}\left(1+r^{2}\right)^{-N} r^{\frac{2 N p-p-2 N}{p}} \mathrm{~d} r} \\
& =C_{N p}^{p-p^{*}}\left(\frac{N-p}{p-1}\right)^{p}(p-1) .
\end{aligned}
$$

Integrating by parts, we have

$$
\int_{0}^{+\infty} \frac{r^{\beta}}{\left(1+r^{2}\right)^{n}} \mathrm{~d} r=\frac{\beta-1}{2 n-\beta-1} \int_{0}^{+\infty} \frac{r^{\beta-2}}{\left(1+r^{2}\right)^{n}} \mathrm{~d} r \quad \text { for } 2 \leq \beta<2 n-1
$$

then

$$
\begin{aligned}
& \frac{K_{1}}{K_{2}}=C_{N p}^{p-p^{*}}\left(\frac{N-p}{p-1}\right)^{p} \frac{(p-1)(N+1)}{N-2 p+1}, \\
& \frac{(N-p) M_{1}}{N M_{2}}=C_{N p}^{p-p^{*}}\left(\frac{N-p}{p-1}\right)^{p}(p-1)
\end{aligned}
$$

since $\frac{N+1}{N-2 p+1}>1$, which implies $\frac{N-p}{N} \frac{M_{1}}{M_{2}}<\frac{K_{1}}{K_{2}}$ holds. Thus,

$$
\sup _{\tau \geq 0} J_{\lambda}\left(\tau u_{\varepsilon}\right)<\frac{S^{\frac{N}{p}}}{2 N\left(Q^{M}\right)^{\frac{N-p}{p}}}=c^{*} .
$$


Proof of Theorem 1.2 Applying Lemmas 2.3, 3.2, and 2.4, we have

$$
0<\beta \leq c=\inf _{h \in \Gamma} \max _{\tau \in[0,1]} J_{\lambda}(h(\tau)) \leq \max _{\tau \geq 0} J_{\lambda}\left(\tau u_{\varepsilon}\right)<c^{*},
$$

and functional $J_{\lambda}(u)$ satisfies the $(P S)_{c}$-condition. Upon an application of mountain pass theorem, we obtain that Problem (1.1) has at least one nontrivial solution $u$. On the other hand, since $J_{\lambda}(u)=J_{\lambda}(|u|)$, then Problem (1.1) has at least one nontrivial nonnegative solution. The proof of Theorem 1.2 is completed.

\section{Proof of Theorem 1.3}

In this section, we study the existence of solution to Problem (1.1) in the case of $\lambda \leq 0$. For the sake of convenience, we replace $\lambda$ by $-\mu$, then Problem (1.1) may be rewritten as the following problem:

$$
\begin{cases}-\Delta_{p} u-\mu|u|^{p-2} u=Q(x)|u|^{p^{*}-2} u+P(x) \frac{|u|^{*}(t)-2}{\left.|x|\right|^{t}}, & x \in \Omega, \\ |\nabla u|^{p-2} \frac{\partial u}{\partial v}=0, & x \in \partial \Omega,\end{cases}
$$

where $P(x)$ changes sign and satisfies $\int_{\Omega} \frac{P(x)}{|x|^{t}} \mathrm{~d} x<0$. Then the corresponding energy functional of problem (4.1) is

$$
\begin{aligned}
J_{\mu}(u) & =\frac{1}{p} \int_{\Omega}|\nabla u|^{p} \mathrm{~d} x-\frac{\mu}{p} \int_{\Omega}|u|^{p} \mathrm{~d} x-\frac{1}{p^{*}} \int_{\Omega} Q(x)|u|^{p^{*}} \mathrm{~d} x-\frac{1}{p^{*}(t)} \int_{\Omega} P(x) \frac{|u|^{p^{*}(t)}}{|x|^{t}} \mathrm{~d} x, \\
u & \in W^{1, p}(\Omega) .
\end{aligned}
$$

Denote $W^{1, p}(\Omega)=V \oplus \operatorname{Span}\{1\}$ with norm $\|u\|_{V}^{p}=\|\nabla v\|_{p}^{p}+|s|^{p}$, where $V=\left\{v \in W^{1, p}(\Omega)\right.$, $\left.\int_{\Omega} v \mathrm{~d} x=0\right\}$, then norms $\|u\|_{V}$ and $\|u\|$ are equivalent.

Lemma 4.1 If there exists a constant $\eta>0$ such that $\|\nabla v\|_{p}=\left(\int_{\Omega}|\nabla v|^{p} \mathrm{~d} x\right)^{\frac{1}{p}} \leq \eta|s|$ for $s \in R, v \in V$, then

$$
\int_{\Omega} \frac{P(x)|v+s|^{p^{*}(t)}}{|x|^{t}} \mathrm{~d} x \leq \frac{|s|^{p^{*}(t)}}{2} \int_{\Omega} \frac{P(x)}{|x|^{t}} \mathrm{~d} x .
$$

Proof The proof is based on $V \hookrightarrow L^{p^{*}(t)}\left(\Omega, \frac{1}{|x|^{t}}\right)$, we omit the details here.

Lemma 4.2 There exist constants $\mu_{0}>0, \rho>0, \beta>0$ such that $J_{\mu}(u) \geq \beta$ for $0 \leq \mu<$ $\mu_{0}$ and $\|u\|_{V}=\rho$. Moreover, $\inf _{\|u\|_{V} \leq \rho} J_{\mu}(u)<0$.

Proof First, we prove that there exist constants $\mu_{0}>0, \rho>0, \beta>0$ such that $J_{\mu}(u) \geq \beta$ for $0 \leq \mu<\mu_{0}$ and $\|u\|_{V}=\rho$, the proof is divided into two cases:

(i) If there exists a constant $\eta>0$ such that $\|\nabla v\|_{p} \leq \eta|s|$, then $|s|^{p} \geq \frac{\|u\|_{V}^{p}}{1+\eta^{p}}$. By Lemma 4.1, we have

$$
J_{\mu}(u) \geq \frac{\alpha\|u\|_{V}^{p^{*}(t)}}{p^{*}(t)\left(1+\eta^{p}\right)^{\frac{p^{*}(t)}{p}}}-\frac{\mu}{p} \int_{\Omega}|u|^{p} \mathrm{~d} x-\frac{1}{p^{*}} \int_{\Omega} Q(x)|u|^{p^{*}} \mathrm{~d} x,
$$

where $\alpha=-\frac{1}{2} \int_{\Omega} \frac{P(x)}{|x|^{t}} \mathrm{~d} x>0$. 
(ii) If there exists a constant $\eta>0$ such that $\|\nabla v\|_{p}>\eta|s|$, then $\|u\|_{V}^{p}=\|\nabla v\|_{p}^{p}+|s|^{p} \leq\left(1+\frac{1}{\eta^{p}}\right)\|\nabla v\|_{p}^{p}$. Using Lemma 2.2 and the norms $\|u\|$ and $\|u\|_{V}$ are equivalent, we see that

$$
\int_{\Omega} P(x) \frac{|u|^{p^{*}(t)}}{|x|^{t}} \mathrm{~d} x \leq C_{3}\|u\|_{V}^{p^{*}(t)} \leq C_{3}\|\nabla v\|_{p}^{p^{*}(t)}\left(1+\frac{1}{\eta^{p}}\right)^{\frac{p^{*}(t)}{p}}
$$

where constant $C_{3}>0$. Consequently,

$$
\begin{aligned}
J_{\mu}(u) \geq & \frac{1}{p}\|\nabla v\|_{p}^{p}-\frac{C_{3}}{p^{*}(t)}\left(1+\frac{1}{\eta^{p}}\right)^{\frac{p^{*}(t)}{p}}\|\nabla v\|_{p}^{p^{*}(t)}-\frac{\mu}{p} \int_{\Omega}|u|^{p} \mathrm{~d} x \\
& -\frac{1}{p^{*}} \int_{\Omega} Q(x)|u|^{p^{*}} \mathrm{~d} x .
\end{aligned}
$$

Choosing $\|u\|_{V}=\rho$ sufficiently small such that $\frac{1}{p}\|\nabla v\|_{p}^{p} \geq \frac{2 C_{3}}{p^{*}(t)}\left(1+\frac{1}{\eta^{p}}\right)^{\frac{p^{*}(t)}{p}}\|\nabla v\|_{p}^{p^{*}(t)}$, we see that

$$
\begin{aligned}
J_{\mu}(u) & \geq \frac{1}{2 p}\|\nabla v\|_{p}^{p}-\frac{\mu}{p} \int_{\Omega}|u|^{p} \mathrm{~d} x-\frac{1}{p^{*}} \int_{\Omega} Q(x)|u|^{p^{*}} \mathrm{~d} x \\
& \geq \frac{\eta^{p}\|u\|_{V}^{p}}{2 p\left(1+\eta^{p}\right)}-\frac{\mu}{p} \int_{\Omega}|u|^{p} \mathrm{~d} x-\frac{1}{p^{*}} \int_{\Omega} Q(x)|u|^{p^{*}} \mathrm{~d} x .
\end{aligned}
$$

Let $k=\min \left\{\frac{\alpha\|u\|_{V}^{p^{*}(t)}}{p^{*}(t)\left(1+\eta^{p}\right)^{\frac{p^{*}(t)}{p}}}, \frac{\eta^{p}\|u\|_{V}^{p}}{2 p\left(1+\eta^{p}\right)}\right\}$. Using (2.4), (4.2), and (4.3), we derive that

$$
J_{\mu}(u) \geq k-\frac{\mu}{p}\|u\|^{p}-\frac{C_{1}}{p^{*}} Q_{M}\|u\|^{p^{*}} .
$$

Noting that $\|u\|$ and $\|u\|_{V}$ are equivalent, thus there exist constants $\mu_{0}>0, \rho>0, \beta>$ 0 such that $J_{\mu}(u) \geq \beta$ for $0 \leq \mu<\mu_{0}$ and $\|u\|_{V}=\rho$.

Next, we prove $\inf _{\|u\|_{V} \leq \rho} J_{\mu}(u)<0$. Since

$$
J_{\mu}(\tau)=-\frac{\mu \tau^{p}}{p}|\Omega|-\frac{\tau^{p^{*}}}{p^{*}} \int_{\Omega} Q(x) \mathrm{d} x-\frac{\tau^{p^{*}(t)}}{p^{*}(t)} \int_{\Omega} P(x) \mathrm{d} x,
$$

by (A1) and (A2), we know that $J_{\lambda}(\tau)<0$ for $\tau>0$ small enough. Consequently, $\inf _{\|u\|_{V} \leq \rho} J_{\mu}(u)<0$.

Taking $\alpha_{\mu}=\inf _{\|u\|_{V} \leq \rho} J_{\mu}(u)$. Next, we give the proof of Theorem 1.3.

Proof of Theorem 1.3 Using Lemma 4.2 and Ekeland's variational principe, we know that there exists a sequence $\left\{u_{n}\right\}$ such that $J_{\mu}\left(u_{n}\right) \rightarrow \alpha_{\mu}, J_{\mu}^{\prime}\left(u_{n}\right) \rightarrow 0$ in $\left(W^{1, p}(\Omega)\right)^{*}$ as $n \rightarrow \infty$. Since $\|u\|_{V} \leq \rho$ and $\bar{B}_{\rho}=\left\{u \in W^{1, p}(\Omega) \mid\|u\|_{V} \leq \rho\right\}$ is closed and convex, there exists a subsequence, still denoted by $\left\{u_{n}\right\}$ and $u \in \bar{B}_{\rho}$, such that

$$
\begin{aligned}
& u_{n} \rightarrow u \quad \text { weakly in } W^{1, p}(\Omega), \\
& u_{n} \rightarrow u \quad \text { weakly in } L^{p^{*}}(\Omega),
\end{aligned}
$$




$$
\begin{array}{ll}
u_{n} \rightarrow u & \text { weakly in } L^{p^{*}(t)}\left(\Omega,|x|^{-t}\right), \\
u_{n} \rightarrow u & \text { strongly in } L^{p}(\Omega), \\
u_{n} \rightarrow u & \text { almost everywhere in } \Omega .
\end{array}
$$

By the Lions concentration-compactness principle [27], we have (2.7) and (2.8) hold. Then, we prove $\mu_{j}=0$ and $v_{j}=0$, where $j \in J$. In fact, we choose $\varepsilon>0$ sufficiently small such that $0 \notin B_{\varepsilon}\left(x_{j}\right)$ and $B_{\varepsilon}\left(x_{i}\right) \cap B_{\varepsilon}\left(x_{j}\right)=\varnothing$ for $i \neq j, i, j \in J$. Let $\phi_{\varepsilon}^{j}(x)$ be a smooth cut-off function centered at $x_{j}$ such that

$$
0 \leq \phi_{\varepsilon}^{j}(x) \leq 1 \quad \text { for }\left|x-x_{j}\right|<\varepsilon, \quad \phi_{\varepsilon}^{j}(x)=\left\{\begin{array}{ll}
1, & \left|x-x_{j}\right| \leq \frac{\varepsilon}{2}, \\
0, & \left|x-x_{j}\right| \geq \varepsilon,
\end{array} \quad \text { and } \quad\left|\nabla \phi_{\varepsilon}^{j}\right| \leq \frac{4}{\varepsilon}\right.
$$

Using the same argument as that in Lemma 2.4, we have

$$
0=\lim _{\varepsilon \rightarrow 0} \lim _{n \rightarrow \infty}\left\langle J_{\mu}^{\prime}\left(u_{n}\right), u_{n} \phi_{\varepsilon}^{j}(x)\right\rangle \geq \mu_{j}-Q\left(x_{j}\right) v_{j}
$$

and from (2.8) we find that $\mu_{j}=0$ or

$$
\begin{aligned}
& \mu_{j} \geq\left(\frac{S^{\frac{p^{*}}{p}}}{Q_{M}}\right)^{\frac{p}{p^{*}-p}}, \quad x_{j} \in \Omega, \\
& \mu_{j} \geq\left(\frac{S^{\frac{p^{*}}{p}}}{2 \frac{p^{*}}{N} Q^{M}}\right)^{\frac{p}{p^{*}-p}}, \quad x_{j} \in \partial \Omega .
\end{aligned}
$$

If $\mu_{j} \neq 0$, it follows from Hölder's inequality and Young's inequality that

$$
\begin{aligned}
0> & \alpha_{\mu}=\lim _{n \rightarrow \infty}\left(J_{\mu}\left(u_{n}\right)-\frac{1}{p^{*}(t)}\left|J_{\mu}^{\prime}\left(u_{n}\right), u_{n}\right\rangle\right) \\
\geq & -\mu \frac{p^{*}(t)-p}{p p^{*}(t)}|\Omega|^{\frac{p^{*}-p}{p^{*}}}\left(\int_{\Omega}|u|^{p^{*}} \mathrm{~d} x\right)^{\frac{p}{p^{*}}}+\frac{p^{*}(t)-p}{p^{*}(t) p} \sum_{j \in J \cup\{0\}} \mu_{j} \\
& +\frac{p^{*}-p^{*}(t)}{p^{*}(t) p^{*}} Q_{m} \int_{\Omega}|u|^{p^{*}} \mathrm{~d} x+\frac{p^{*}-p^{*}(t)}{p^{*}(t) p^{*}} \sum_{j \in J \cup\{0\}} Q\left(x_{j}\right) v_{j} \\
\geq & -\left(\mu \frac{p^{*}(t)-p}{p p^{*}(t)}\right)^{\frac{p^{*}}{p^{*}-p}}|\Omega|\left(\frac{p^{*}-p^{*}(t)}{p^{*}(t) p^{*}} Q_{m}\right)^{-\frac{p}{p^{*}-p}}+\frac{p^{*}(t)-p}{p^{*}(t) p} \sum_{j \in J \cup\{0\}} \mu_{j} \\
> & 0
\end{aligned}
$$

for $\mu<\mu^{*}=\min \left\{\mu_{0}, \mu_{1}\right\}$, where $\mu_{1}=\min \left\{\left(\frac{p\left(p^{*}-p^{*}(t)\right) Q_{m}}{p^{*}\left(p^{*}(t)-p\right)|\Omega|^{\frac{p^{*}-p}{p}}}\right)^{\frac{p}{p^{*}}} \frac{S}{Q_{M}^{\frac{p}{p^{*}}}},\left(\frac{p\left(p^{*}-p^{*}(t)\right) Q_{m}}{p^{*}\left(p^{*}(t)-p\right)|\Omega|^{\frac{p^{*}-p}{p}}}\right)^{\frac{p}{p^{*}}} \times\right.$

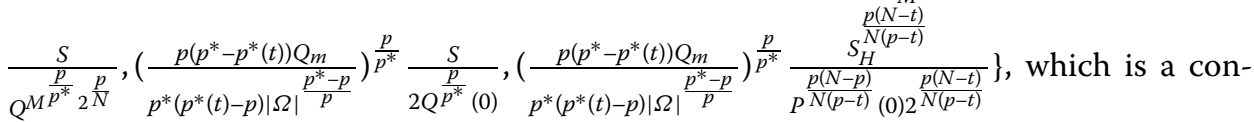
tradiction. Hence, $\mu_{j}=v_{j}=0$. 
Next, we prove $\mu_{0}=\nu_{0}=\gamma_{0}=0$. Let $\varepsilon>0$ be small enough such that $x_{j} \notin B_{\varepsilon}(0), j \in J$, choose a smooth cut-off function $\phi_{\varepsilon}^{0}(x)$ such that

$$
0 \leq \phi_{\varepsilon}^{0}(x) \leq 1 \quad \text { for }|x|<\varepsilon, \quad \phi_{\varepsilon}^{0}(x)=\left\{\begin{array}{ll}
1, & |x| \leq \frac{\varepsilon}{2}, \\
0, & |x| \geq \varepsilon,
\end{array} \quad \text { and } \quad\left|\nabla \phi_{\varepsilon}^{0}\right| \leq \frac{4}{\varepsilon}\right.
$$

Similarly, we get $\mu_{0} \leq Q(0) v_{0}+P(0) \gamma_{0}$. If $\mu_{0} \neq 0$, we have

$$
\frac{\mu_{0}}{2} \leq Q(0) v_{0} \quad \text { or } \quad \frac{\mu_{0}}{2} \leq P(0) \gamma_{0}
$$

then using (2.8), we can deduce that

$$
\mu_{0} \geq\left(\frac{S^{\frac{p^{*}}{p}}}{2 Q(0) 2^{\frac{p^{*}}{N}}}\right)^{\frac{p}{p^{*}-p}} \quad \text { or } \quad \mu_{0} \geq\left(\frac{S_{H}^{\frac{p^{*}(t)}{p}}}{2 P(0) 2^{\frac{p-t}{N-p}}}\right)^{\frac{p}{p^{*}(t)-p}} .
$$

Bringing the above inequalities into (4.4), we see that

$$
0>\alpha_{\mu} \geq-\left(\mu \frac{p^{*}(t)-p}{p p^{*}(t)}\right)^{\frac{p^{*}}{p^{*}-p}}|\Omega|\left(\frac{p^{*}-p^{*}(t)}{p^{*}(t) p^{*}} Q_{m}\right)^{-\frac{p}{p^{*}-p}}+\frac{p^{*}(t)-p}{p^{*}(t) p} \mu_{0}>0
$$

for $\mu<\mu^{*}$, which is a contradiction. Hence, $\mu_{0}=v_{0}=\gamma_{0}=0$ and $u_{n} \rightarrow u$ strongly in $W^{1, p}(\Omega), \frac{\left|u_{n}\right|^{* *(t)}}{\left.|x|\right|^{t}} \rightarrow \frac{|u|^{* *(t)}}{|x|^{t}}$ strongly in $L^{p^{*}(t)}(\Omega)$. So $u$ is a solution of Problem (4.1) and $\alpha_{\mu}=J_{\mu}(u)$. Since $J_{\mu}(u)=J_{\mu}(|u|)$, then Problem (4.1) has at least one nontrivial nonnegative solution. The proof of Theorem 1.3 is completed.

\section{Acknowledgements}

The authors would like to thank the editor and the referees for their valuable comments and suggestions, which improved the quality of our manuscript.

\section{Funding}

This work is supported by the National Natural Science Foundation of China (Grant No. 11601122,11847081), Foundation of Henan Educational Committee (Grant No. 19A110007), and the Fundamental Research Funds for the Henan Provincial Colleges and Universities in Henan University of Technology (Grant No. 2018QNJH12).

\section{Availability of data and materials}

Data sharing not applicable to this article as no datasets were generated or analysed during the current study.

\section{Competing interests}

The authors declare that they have no competing interests.

\section{Authors' contributions}

The authors contributed equally to the writing of this paper. All authors read and approved the final manuscript.

\section{Publisher's Note}

Springer Nature remains neutral with regard to jurisdictional claims in published maps and institutional affiliations.

Received: 30 September 2019 Accepted: 3 March 2020 Published online: 11 March 2020

\section{References}

1. Comte, M., Knaap, M.C.: Existence of solutions of elliptic equations involving critical Sobolev exponents with Neumann boundary conditions in general domains. Differ. Integral Equ. 6, 1132-1146 (1991)

2. Adimurthi, Yadava, S.L.: Existence of a nonradial positive solution for the critical exponent with Neumann boundary condition. J. Differ. Equ. 104, 298-306 (1993)

3. Chabrowski, J., Willem, M.: Least energy solutions of a critical Neumann problem with a weight. Calc. Var. Partial Differ. Equ. 15, 421-431 (2002) 
4. Chabrowski, J.: On the Neumann problem with multiple critical nonlinearities. Complex Var. Elliptic Equ. 55, 501-524 (2010)

5. Shang, Y.Y., Tang, C.L.: Positive solutions for Neumann elliptic problems involving critical Hardy-Sobolev exponent with boundary singularities. Nonlinear Anal., Theory Methods Appl. 70, 1302-1320 (2009)

6. Cao, D.M., Noussair, E.S.: The effect of geometry of the domain boundary in an elliptic Neumann problem. Adv. Differ. Equ. 6, 931-958 (2001)

7. Chabrowski, J.: On the Neumann problem with the Hardy-Sobolev potential. Ann. Mat. Pura Appl. 186, 703-719 (2007)

8. Garcia-Azorero, J., Peral, I., Rossi, J.D.: A convex-concave problem with a nonlinear boundary condition. J. Differ. Equ. $198,91-128(2004)$

9. Hashizume, M.: Asymptotic behavior of the least-energy solutions of a semilinear elliptic equation with the Hardy-Sobolev critical exponent. J. Differ. Equ. 262, 3107-3131 (2017)

10. Cao, D.M., Yan, S.S.: Infinitely many solutions for an elliptic Neumann problem involving critical Sobolev growth J. Differ. Equ. 251, 1389-1414 (2011)

11. Han, P.G., Liu, Z.X.: Positive solutions for elliptic equations involving critical Sobolev exponents and Hardy terms with Neumann boundary conditions. Nonlinear Anal., Theory Methods Appl. 55, 167-186 (2003)

12. Yang, H.T., Chen, J.H.: A result on Hardy-Sobolev critical elliptic equations with boundary singularities. Commun. Pure Appl. Anal. 61, 191-201 (2007)

13. Abreu, E.A.M., do Ó, J.M., Medeiros, E.S.: Multiplicity of positive solutions for a class of quasilinear nonhomogeneous Neumann problems. Nonlinear Anal., Theory Methods Appl. 60, 1443-1471 (2005)

14. Deng, Y.B., Jin, L.Y.: Multiple positive solutions for a quasilinear nonhomogeneous Neumann problems with critical Hardy exponents. Nonlinear Anal.. Theory Methods Appl. 67, 3261-3275 (2007)

15. Li, Y.X., Xia, S.X.: Existence of multiple solutions for a quasilinear Neumann problem with critical exponent. Bound. Value Probl. 2018, Article ID 66 (2018)

16. Filippucci, R., Pucci, P., Robert, F.: On a p-Laplace equation with multiple critical nonlinearities. J. Math. Pures Appl. 91 , 156-177 (2009)

17. Li, Y.Y., Guo, Q.Q., Niu, P.C.: The existence of solutions for quasilinear elliptic problems with combined critical Sobolev-Hardy terms. J. Math. Anal. Appl. 388, 525-538 (2012)

18. Bhakta, M.: Infinitely many sign-changing solutions of an elliptic problem involving critical Sobolev and Hardy-Sobolev exponent. Proc. Indian Acad. Sci. Math. Sci. 127, 337-347 (2017)

19. Chen, G.W., Ma, S.W.: On the quasilinear elliptic problem with a Hardy-Sobolev critical exponent. Dyn. Partial Differ. Equ. 8(3), 225-237 (2011)

20. Ghoussoub, N., Yuan, C.: Multiple solutions for quasilinear PDEs involving critical Sobolev and Hardy exponents. Trans, Am. Math. Soc. 352, 5703-5743 (2000)

21. Zhao, P.H., Zhao, J.H.: Existence of infinitely many weak solutions for the $p$-Laplacian with nonlinear boundary conditions. Nonlinear Anal., Theory Methods Appl. 69, 1343-1355 (2008)

22. Alves, C.O., Hamidi, A.E.: Nehari manifold and existence of positive solutions to a class of quasilinear problems, Nonlinear Anal., Theory Methods Appl. 60, 611-624 (2005)

23. Cingolani, S., Vannella, G.: Multiple positive solutions for a critical quasilinear equation via Morse theory. Ann. Inst. Henri Poincaré, Anal. Non Linéaire 26, 397-413 (2009)

24. Li, Y.X., Gao, W.G.: Existence of multiple solutions for quasilinear elliptic equation with critical Sobolev-Hardy terms. Math. Methods Appl. Sci. 38, 145-154 (2015)

25. Perera, K., Zou, W.M.: p-Laplacian problems involving critical Hardy-Sobolev exponents. Nonlinear Differ. Equ. Appl. 25(25), 1-16 (2018)

26. Xiang, C.L.: Asymptotic behaviors of solutions to quasilinear elliptic equations with critical Sobolev growth and Hardy potential. J. Differ. Equ. 259, 3929-3954 (2015)

27. Lions, P.L.: The concentration-compactness principle in the calculus of variations. The limit case Part II. Rev. Mat. Iberoam. 1, 145-201 (1985)

\section{Submit your manuscript to a SpringerOpen ${ }^{\circ}$ journal and benefit from:}

- Convenient online submission

- Rigorous peer review

- Open access: articles freely available online

- High visibility within the field

- Retaining the copyright to your article

Submit your next manuscript at $\gg$ springeropen.com 